\title{
A Computational Comparison of Evolutionary Algorithms for Water Resource Planning for Agricultural and Environmental Purposes
}

\author{
James Montgomery \\ School of Technology, Environments and Design \\ University of Tasmania \\ Hobart, Australia \\ james.montgomery@utas.edu.au \\ Marcus Randall \\ Bond Business School \\ Bond University \\ Gold Coast, Australia \\ mrandall@bond.edu.au
}

\author{
Andrew Fitzgerald* \\ Department of Primary Industries, Parks, Water and Environment \\ Tasmanian Government \\ Hobart, Australia \\ andrewf@utas.edu.au
}

Andrew Lewis

School of Information and Communication Technology

Griffith University

Nathan, Australia

a.lewis@griffith.edu.au

\begin{abstract}
The use of water resources for agricultural purposes, particularly in arid and semi-arid regions, is a matter of increasing concern across the world. Optimisation techniques can play an important role in improving the allocation of land to different crops, based on a utility function (such as net revenue) and the water resources needed to support these. Recent work proposed a model formulation for an agricultural region in the Murrumbidgee Irrigation Area of the Murray-Darling River basin in Australia, and found that the well-known NSGA-II technique could produce sensible crop mixes while preserving ground and surface water for environmental purposes. In the present study we apply Differential Evolution using two different solution representations, one of which explores the restricted space in which no land is left fallow. The results improve on those of the prior NSGA-II and demonstrate that a combination of solution representations allows Differential Evolution to more thoroughly explore the multiobjective space of profit versus environment.
\end{abstract}

Index Terms-water resource management, crop planning, differential evolution, NSGA-II

\section{INTRODUCTION}

Crop planning, particularly in arid and semi-arid regions (such as Australia) is an ongoing issue due to climate change and increasing populations with specific food consumption patterns [1]. Given that there are limited resources such as arable land and water, the problem of determining which crops to plant, and when, naturally becomes a decision making and optimisation one. As such, there have been some attempts to formulate appropriate mathematical models and develop algorithms to determine optimal planting strategies. While these could focus exclusively on trying to maximise the profit (referred to as net revenue), a competing but equally important

\footnotetext{
* This research was principally conducted while Andrew Fitzgerald was a Masters of Information Technology and Systems student at the University of Tasmania. The Tasmanian Government is not affiliated with the present work.
}

objective is to satisfy demands of environmental sustainability. This is particularly in terms of the amount of water that is drawn from underground aquifers and the surface (i.e., river systems).

This multiobjective problem has been studied by Xevi and Khan [2] and further refined by the work of Lewis and Randall [3]. The latter used the tool known as the Non-dominated Sorting Genetic Algorithm-II (NSGA-II) [4]. To better understand and explore the search space of this problem we apply an alternative algorithm, Differential Evolution (DE) [5], [6]. Specifically, we investigate how such a continuous solver may be applied to this discrete problem using two alternative solution representations, and the potential advantages that may be gained. The purpose of the present study is not to identify the single 'best' algorithm for this problem-as the problem definition is still an active area of investigation this would be premature-but to investigate how a continuous solver such as DE can be applied. The application of a broader range of standard evolutionary algorithms will be the subject of future work.

The remainder of this paper is organised as follows. Section II describes the previous work done by Xevi and Khan [2] and Lewis and Randall [3] on the development of the model and the optimisation algorithms for this problem. Section III explores how DE can be adapted to suit this problem in terms of the representation schemes that it can use. Section IV analyses the performance of DE with respect to the previous solutions generated by NSGA-II [3]. We also describe how these new solutions relate to the crop planning context and examine the new insights they reveal. Section $\mathrm{V}$ discusses the overall implications of this work and also looks forward to the projects and lines of enquiry that it opens up. 


\section{Water Management And Previous COMPUTATIONAL APPROACHES}

The problem considered in this work is based on a common agricultural scenario: for the upcoming year, a variety of crops are to be allocated space within a farming area and are to be supplied with water from natural rainfall, pumped groundwater, and water pumped from one or more adjacent waterways. The original model of this problem was given by Xevi and Khan [2] and later refined by Lewis and Randall [3]. The latter model is the one used herein, and for convenience is reproduced in Equations 1-7. Extensive explanation of it may be found in that paper.

$$
\begin{aligned}
\text { Maximise } N R= & \sum_{c=1}^{C} T C I(c) \times X(c)-C_{w} \\
& \times \sum_{m=1}^{M}\left(\left(\sum_{c=1}^{C} W R E Q(c, m) \times X(c)\right)\right. \\
& -P(m))-C_{p} \times \sum_{m=1}^{M} P(m) \\
& -\sum_{c=1}^{C} V \operatorname{cost}(c) \times X(c)
\end{aligned}
$$

$$
\text { Minimise } \begin{aligned}
E N= & \sum_{m=1}^{M}\left(T e n v_{-} f(m)-E n v_{-} f(m)\right) \\
& \times\left[E n v_{\_} f(m)<T e n v_{\_} f(m)\right]
\end{aligned}
$$

s.t.,

$$
\begin{gathered}
\sum_{m=1}^{M} P(m) \leq 50 \mathrm{GL} \\
\sum_{c=1}^{C} X(c) \leq \text { Tarea } \\
X(c) \leq Y(c) \quad 1 \leq c \leq C \\
\operatorname{Allocation}(m)=\operatorname{Inflow}(m)-\text { Env_f }(m) \\
P(m)=\left(\sum_{c=1}^{C} W R E Q(c, m) \times X(c)\right)-\operatorname{Allocation}(m)
\end{gathered}
$$

Where:

$N R$ is the net revenue,

$C$ is the number of crops,

$M$ is the number of months, i.e., 12 ,

$T C I(c)$ is the total crop income for crop $c$,

$X(c)$ is a decision variable for the area of crop $c$ (hectares), $W R E Q(c, m)$ is the water required by crop $c$ in month $m$ (ML),
$C_{w}$ is total cost of water per unit volume ( $\left.\$ / \mathrm{ML}\right)$,

$C_{p}$ is the cost of groundwater pumping and delivery (\$/ML), $V \operatorname{cost}(c)$ are all other costs associated with crop $c$,

$T_{\text {Area }}$ is the total cropping area available,

$E n v_{-} f(m)$ is a decision variable for the environmental flow in month $m$,

$E N$ is the deficit in environmental flow,

Tenv_f $f(m)$ is the target environmental flow in month $m$,

$P(m)$ is the groundwater pumped in month $m$,

$Y(c)$ is the maximum allowable area for crop $c$,

Inflow $(m)$ is the total surface (river) water available in month $m$, and

Allocation $(m)$ is the amount of surface water available for irrigation of crops in month $m$.

A solution to the problem comprises the integer-valued area (in hectares) to be allocated to each crop (zero if that crop is not to be planted) and the amount of incoming water to be left to flow downstream each month. Allowing greater environmental flows may require that crop needs are met with more expensive pumped water. The problem can be posed as multiobjective, with the goals of maximising the (estimated) net revenue $(N R)$ from the crops planted while minimising the deficit between the target environmental flow in downstream waterways (required to maintain river health) and the actual environmental flow, referred to hereafter as the environmental flow deficit $(E N)$. The model's chief simplification of planning for a single year will be addressed in later research.

Different problem instances are derived from three components: the alternative crops (and their anticipated profits, costs and water needs throughout the year); details of the agricultural area, such as its size and the local costs of pumping groundwater; and forecasts for rainfall (which also modifies the amount of water needed by crops and costs for pumping groundwater). A common application would fix the crop options and region, and derive alternative instances for different rainfall forecasts, which is the approach taken here.

The particular region considered here has an area of 121,808 ha and is located in the the Murrumbidgee Irrigation Area (MIA), a 660,000 ha area in New South Wales, Australia [2], [7]. The model incorporates a constraint reflecting changes in the administration of the MIA since 2005. On 1 October 2006, the Water Sharing Plan for the Lower Murrumbidgee Groundwater Services (the Plan) commenced, meaning that groundwater was then managed under the Water Management Act 2000 [3]. These regulations set a limit on the annual extraction of groundwater of 270 GL in the MIA, where the research is modelled [3], [8].

Three problem instances are considered, described in detail by Lewis and Randall [3], which model 16 alternative crops and three different rainfall scenarios of 'dry', 'average' and 'wet'. Five of the crops have constraints on their maximum cropping area as they are highly lucrative (making them attractive to an optimisation algorithm), yet the market would not be able to accept the maximum volumes that could be generated by this region. For example, one solution without these bounds yielded 7.5 million tonnes of winter vegetables, 
which is over three times the annual consumption in Australia. One must also bear in mind that the MIA is a relatively small growing region.

An important crop that the authors introduced into their revised model was cotton. It was found that across dry, average and wet scenarios this crop dramatically increased net revenue, but at the expense of large water usage. Their revised model also produced comparable solutions (in terms of net revenue) to the previous work of Xevi and Khan [2], but with minimal expensive groundwater usage. Taking this idea further, they also allowed for seasonal variation in the monthly water targets. Overall, it was shown that their multiobjective approach could produce a family of useful solutions for farmers and regional planners for average and dry conditions. However, under wet conditions only one non-dominated solution was found consisting of a large area of cotton, less of rice, some canola, fruit and vegetables yielding a large net revenue of \$223 million.

\section{ApPlying DifFERENTIAL EVOLUtion}

As solutions to this problem are pseudo-continuous it may be approached using continuous solvers (working with integer-valued solution vectors) such as Differential Evolution (DE) [9] and Particle Swarm Optimisation (PSO) [10]. The present work uses DE as an exemplar continuous solver. The two alternative solution representations described in Section III-A likely impact the solutions generated far more than the solution generation mechanism employed.

A multiobjective DE algorithm is derived using a similar approach to Montgomery, Randall and Lewis in their DE for RFID antenna design [11]: a DE/rand/1/bin algorithm is used to generate new solutions from the current population, then the next generation is selected by applying the nondominated sorting algorithm from NSGA-II to the union of these solution sets. In the present work, feasible solutions are compared using standard Pareto-dominance rules, any feasible solution dominates any infeasible solution, while infeasible solutions are compared based on the amount they violate the pumped water constraint to provide some selection pressure toward feasible space. ${ }^{1}$ The population size-hence, solution archive size - is 100, and the algorithm is executed for 2000 iterations (200,000 function evaluations). Appropriate values of difference vector scale $F$ and crossover probability $\mathrm{Cr}$ are considered as part of this work.

\section{A. Solution Representations}

Two alternative solution representations were examined, one that gives access to the entire search space and another than restricts the search to those solutions in which the entire area is allocated to crops, with no fallow land. ${ }^{2}$

\footnotetext{
${ }^{1}$ This rule, which ignores over-planting, was determined after an initial investigation of the causes of infeasibility in solutions.

${ }^{2}$ It is acknowledged that, since the cultivation of annual crops (as opposed to perennials) will not occupy farmland for the entire year, reservation of an area for such a crop will imply the land lies fallow for some part of the year. This is an issue to be considered in future work.
}

Original DE solution: \begin{tabular}{|l|l|l|l|l|}
0.2 & 0.6 & 0.8 & 0.7 & 0.3 \\
\hline
\end{tabular}

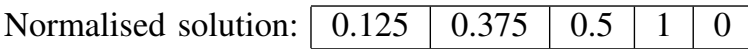

Allocate bounded crops: \begin{tabular}{|l|l|l|l|l|l} 
& $?$ & $?$ & $?$ & 10 & 0 \\
\hline
\end{tabular}

Allocate other crops: \begin{tabular}{|l|l|l|l|l|l|}
\hline 11 & 34 & 45 & 10 & 0 & 0 ha remains \\
\hline
\end{tabular}

Fig. 1. Illustration of decoding the proportional representation for a 100 ha area; the last two crops are bounded at $10 \mathrm{ha}$.

The most straightforward way to encode a solution to this problem is as a vector of $C+12$ integer values (i.e., 28 in the current problem) corresponding to the areas allocated to the $C$ crops and environmental flows for each month of the year. The ranges of 'unbounded' crops are 0 to the size of the farming land $(121,808 \mathrm{ha})$, while bounded crops are restricted to 0 to their nominated maximum. Environmental flow variables range between 0 and the target environmental flow. We refer to this solution encoding as the naive representation. ${ }^{3}$

In this constrained space it is clear that infeasible solutions are a risk. Considering crop area alone, approximately $90 \%$ of the solution space represents infeasible solutions in which more area is allocated than is available. As a means of eliminating this particular cause of infeasibilty, an alternative representation (referred to as the proportional representation hereafter), was also devised in which crop areas are encoded as real numbers in the range $[0,1]$. The representation encodes environmental flows for each month in the same way as the naive. Such a solution is decoded to produce a crop allocation in hectares as follows:

- Bounded crops are treated as binary variables: if their solution value is $\geq 0.5$ then they are allocated their maximum, otherwise they are not planted.

- Unbounded crops are then allocated space from the remaining unallocated area in direct proportion to their normalised solution value.

Figure 1 illustrates this process for a fictitious problem with 100 ha available space and five crops, the last two of which are bounded at 10 ha each. As the fourth (bounded) crop's value is above 0.5 it is allocated its maximum area of 10 ha, while the fifth (bounded) crop is allocated nothing. Finally crops 13 are allocated space from the remaining 90 ha in proportion to their relative magnitude.

\section{B. Initial Solution Generation}

Initial solutions for the naive representation are created by the following steps:

1) Generate $C$ uniform random values $r_{c}$ in $[0,1]$.

2) Allocate each bounded crop $c$ with $r_{c} \geq 0.5$ its maximum area.

3) Normalise the $r_{c}$ values for all remaining crops with $r_{c} \geq$ 0.5 , then allocate those crops space from the remaining area in proportion to their normalised $r_{c}$ values.

\footnotetext{
${ }^{3}$ The label 'naive' is not intended to be pejorative, merely to indicate that this representation only applies the grossest level of constraints, without considering the impact of one crop's land usage on another's.
} 
4) Generate 12 randomised integer values in the range $\left[0, T e n v \_f\right]$ to set the solution's environmental flows.

Initial solutions for the proportional representation are generated using steps 1 and 4 only.

\section{Sensitivity Analysis}

The DE/rand/1/bin was executed on the three problem instances with both solution representations using combinations of the DE control parameters $\mathrm{Cr} \in\{0.1,0.5,0.9\}$ and $F \in\{0.3,0.8\}$. Each combination was run for 2,000 iterations (200,000 function evaluations) with 21 randomised trials performed. Performance was measured by the number of feasible solutions generated and the hypervolume (HV) [12] of the final solution set produced. Hypervolume was calculated within the region bounded by $\left[0,2.88 \times 10^{8}\right]$ in the $N R$ objective - the upper bound being a little above the highest observed-and $\left[0,1.2 \times 10^{6}\right]$ in the $E N$ objective (the upper bound represents no environmental flow and hence a maximum flow deficit). Values of the $E N$ objective were scaled by 300 so that the spread of values, and hence their contribution to $\mathrm{HV}$, was of a similar magnitude to those in the $N R$ objective. Reported values are expressed as a percentage of the rectangular region defined by those bounds.

Figure 2 presents box plots of the proportion of feasible solutions produced for each instance-representation-parameter combination, showing results for the naive representation in the first row and proportional in the second. Mann-Whitney tests show that most of the observed differences are statistically significant at the $1 \%$ level. For high $\mathrm{Cr}$, naive shows a clear downward trend with increasing $F$, suggesting that the larger moves in solution space are more likely to produce infeasible children. The proportional representation tends to produce fewer feasible solutions, but performs best in this regard for either high or low $\mathrm{Cr}$, with lower $\mathrm{F}$ also improving the number of feasible solutions.

Figure 3 presents box plots of the HV achieved by each experimental combination. The outcomes of two-tailed MannWhitney tests are mixed, with many of the observed differences statistically significant, but not all. Results for the naive representation suggest a slight benefit from higher $\mathrm{Cr}$ values and the higher value of $F$. In terms of median performance, $C r=0.9, F=0.3$ and $C r=0.5, F=0.8$ tend to produce the best outcomes (on dry and average).

$\mathrm{HV}$ results for the proportional representation show a clearer trend of increasing performance as $\mathrm{Cr}$ increases, with the best results produced when $C r=0.9, F=0.3$. On the dry and average instances, the proportional representation's HV tends to be markedly lower than that achieved using the naive representation, which is due to the shape of the Pareto front that can be achieved when the algorithm is required to allocate all available space. Figure 4, presented in the discussion in Section IV, illustrates the difference.

Subsequent analyses consider a single $C r, F$ combination for each representation. With the naive representation both $(0.9,0.3)$ and $(0.5,0.8)$ produce good HV results and the dry and average instances, with $(0.5,0.8)$ typically better on dry (statistically significant at the 5\% level), while the slightly better performance of $(0.9,0.3)$ on the average instance is not statistically significant $(p=.14)$. Consequently, despite the lower number of feasible solutions produced by $(0.5,0.8)$ under dry and average conditions, it is used in later analyses of the naive representation. Using the proportional representation, the combination $(0.9,0.3)$ outperforms all others in HV performance (statistically significant at the $1 \%$ level) and is one of the best for feasible solutions, so is selected for later analysis. In the analyses that follow, data was collected about the child and archive populations at iteration 1 and for every $50^{\text {th }}$ iteration.

\section{Causes of Infeasibility}

For the naive representation with $C r=0.5, F=0.8$, approximately $59 \%$ of solutions produced for the dry and average instances are infeasible. The principle cause of infeasibility (more than $98 \%$ of cases) on these instances is overpumping, which occurs when the allocation of crops (and hence their water needs) and environmental flows have been selected such that too much water needs to be extracted from groundwater sources. While the degree of constraint violation varied, it was frequently more than $88 \mathrm{GL}$.

Overplanting was a moderate cause, with approximately $22 \%$ of infeasible solutions (13\% of all solutions) for the dry instance and $31 \%$ (18\% overall) for the average instance due to allocating more crop area than available. This would, in turn, lead to high water needs that can lead to overpumping. In the wet instance, where water is abundant, overplanting is the main cause of infeasibility, but tends to represent only $6 \%$ of generated solutions.

Significantly, this indicates that overplanting, an obvious risk with the naive representation, was not as significant a problem as crop selection, with the algorithm able to find feasible space with regard to this constraint. Indeed, across the final population (which represents differing trade-offs of profit versus environmental benefit) the typical amount of fallow land was approximately $28 \%$ in the dry instance and $15 \%$ in the average instance $(0 \%$ in the more tractable wet instance).

The sole cause of infeasible solutions when using the proportional representation is overpumping, as it cannot encode a solution with more or less than the total amount of space allocated. Using this representation and with $\mathrm{Cr}=0.9, \mathrm{~F}=0.3$, the algorithm tended to produce $65 \%, 54 \%$ and $0 \%$ infeasible solutions for the dry, average and wet instances, respectively. The typical amount of overpumping was approximately $30 \mathrm{GL}$, which is lower than the constraint violation when using naive, suggesting that this more constrained search space allows the algorithm to better deal with (the remaining) constraints.

\section{COMPARING DE WITH Prior ApProACHES}

Figure 4 presents the median and best attainment surfaces in terms of $\mathrm{HV}$ produced by DE with the selected parameter settings $(C r=0.5, F=0.8$ for naive $C r=0.9, F=0.3$ for proportional) as well as the attainment surface produced by Lewis and Randall's [3] NSGA-II. Considering the DE results 


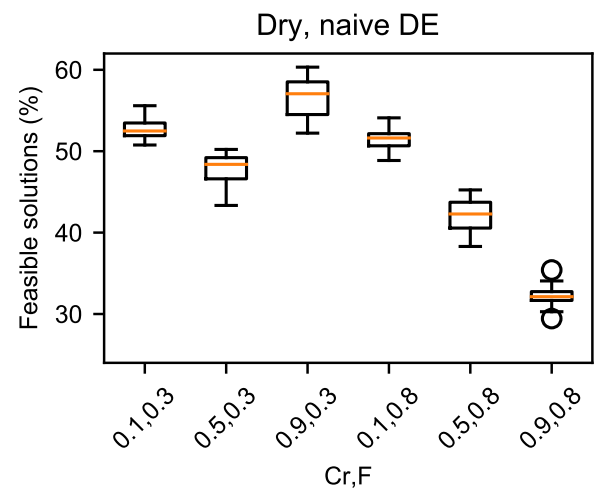

Dry, proportional DE

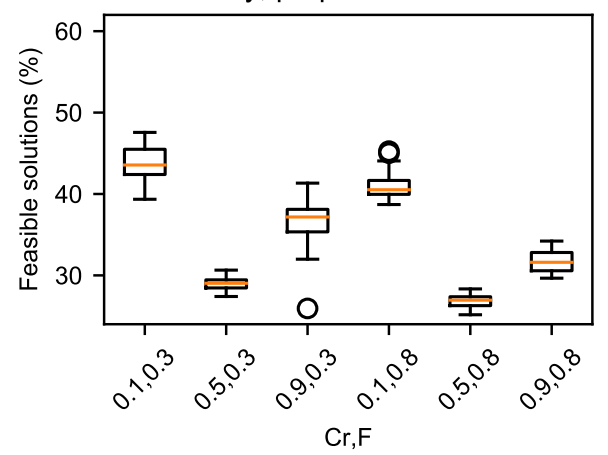

Average, naive DE

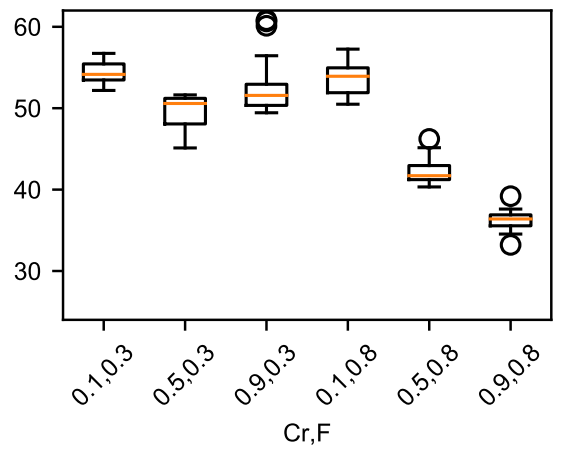

Average, proportional DE

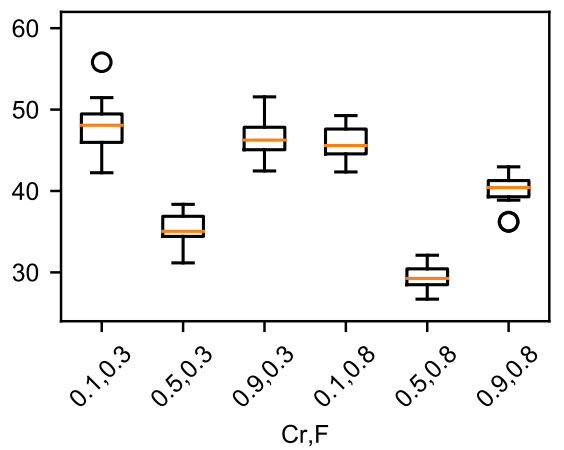

Wet, naive DE

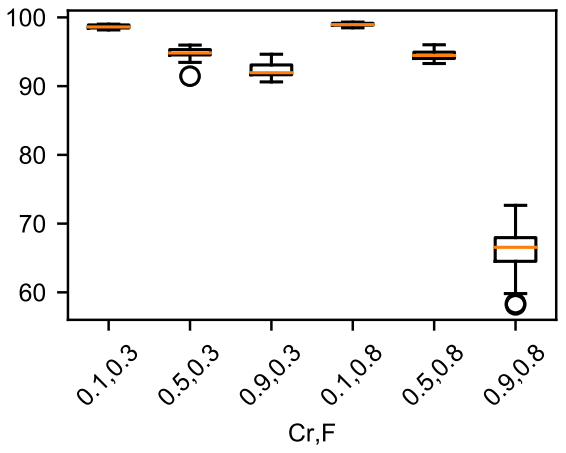

Wet, proportional DE

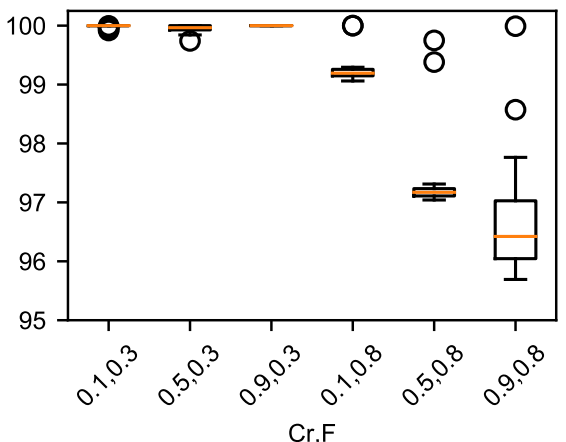

Fig. 2. Proportion of feasible solutions produced for different parameter settings and solution representation, by instance. Plot ranges differ by instance and representation to allow detail to be seen
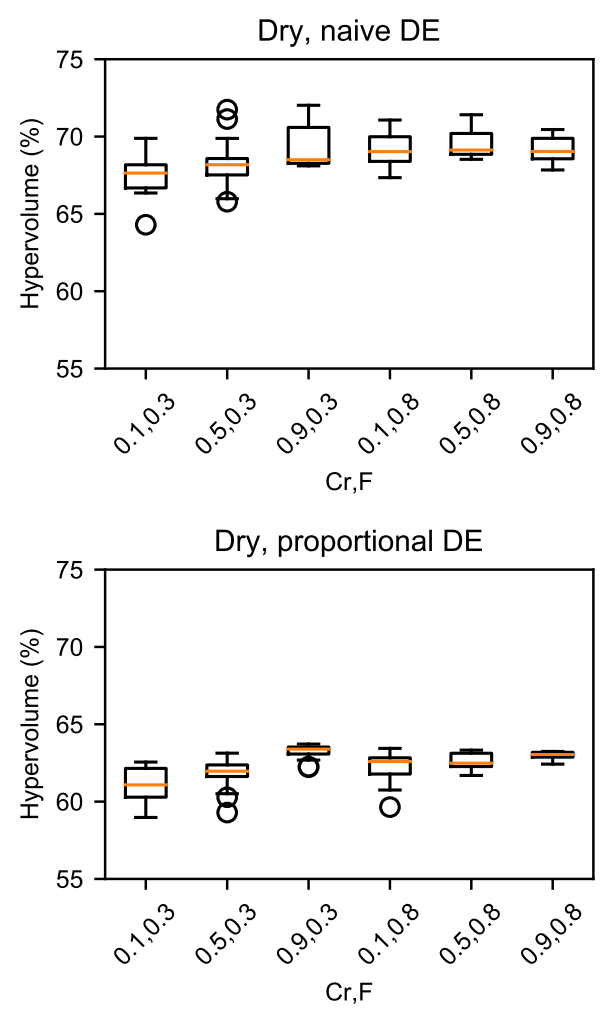

Average, naive DE

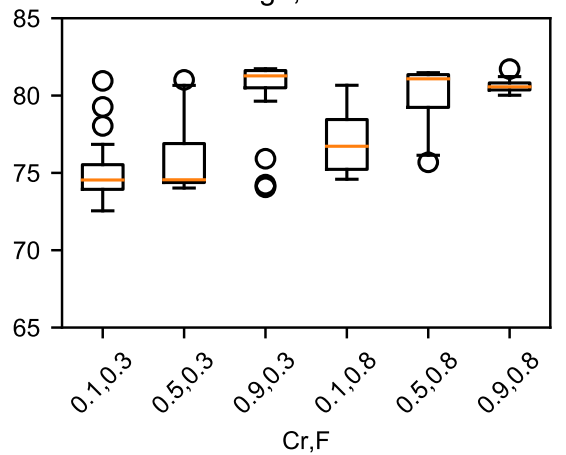

Average, proportional DE

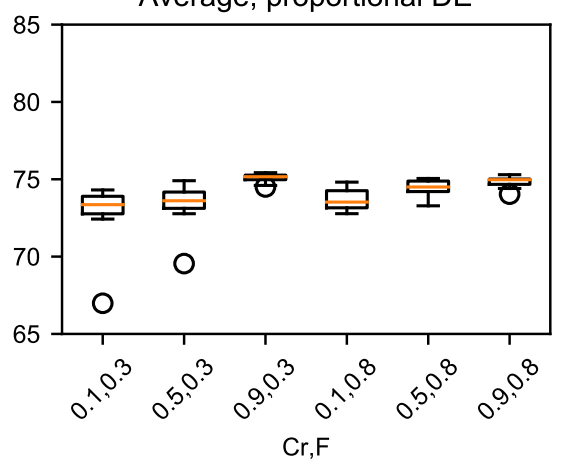

Wet, naive DE

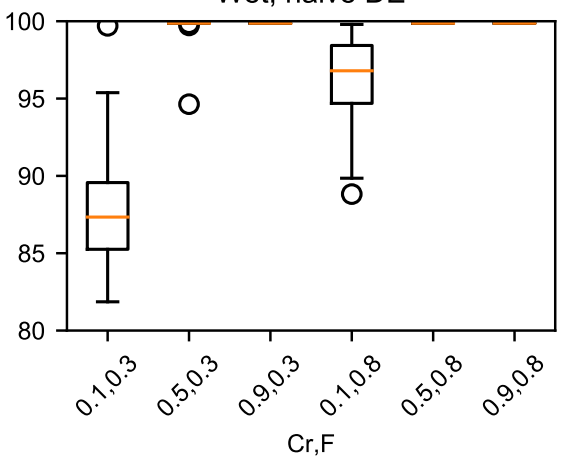

Wet, proportional DE

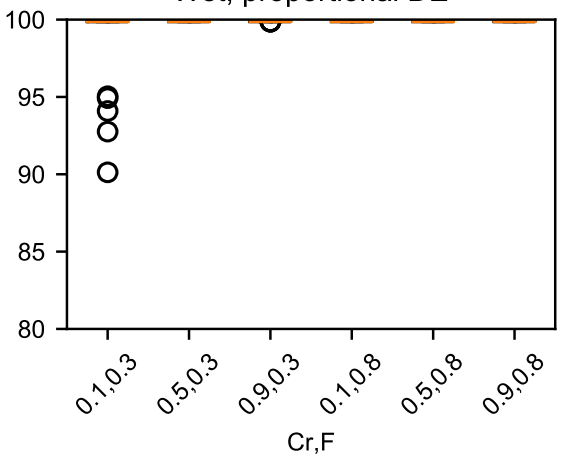

Fig. 3. HV of solution sets produced by different parameter settings and solution representation, by instance. Plot ranges differ by instance to allow detail to be seen 


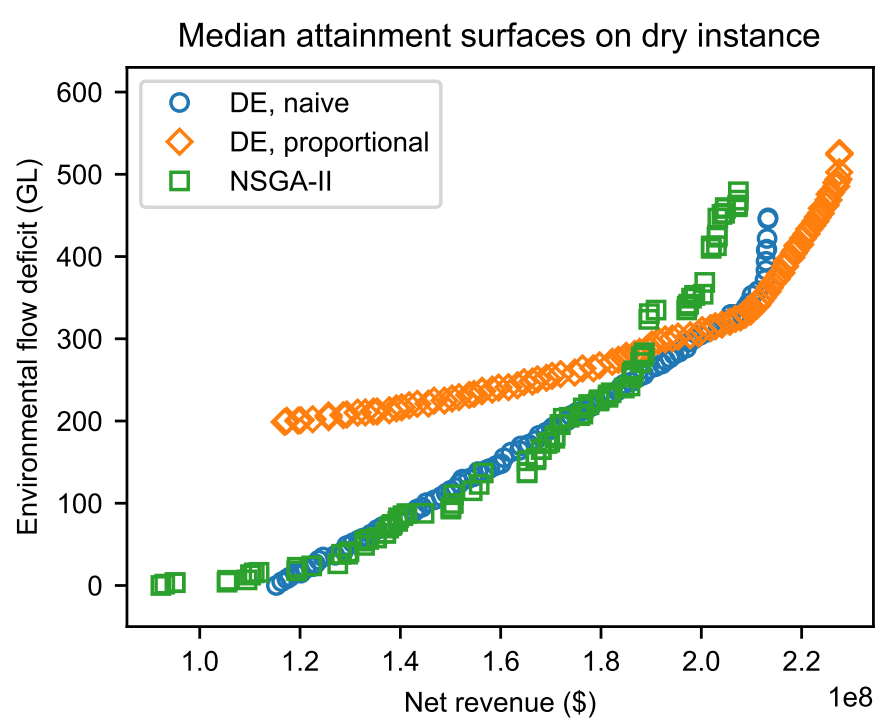

Median attainment surfaces on average instance
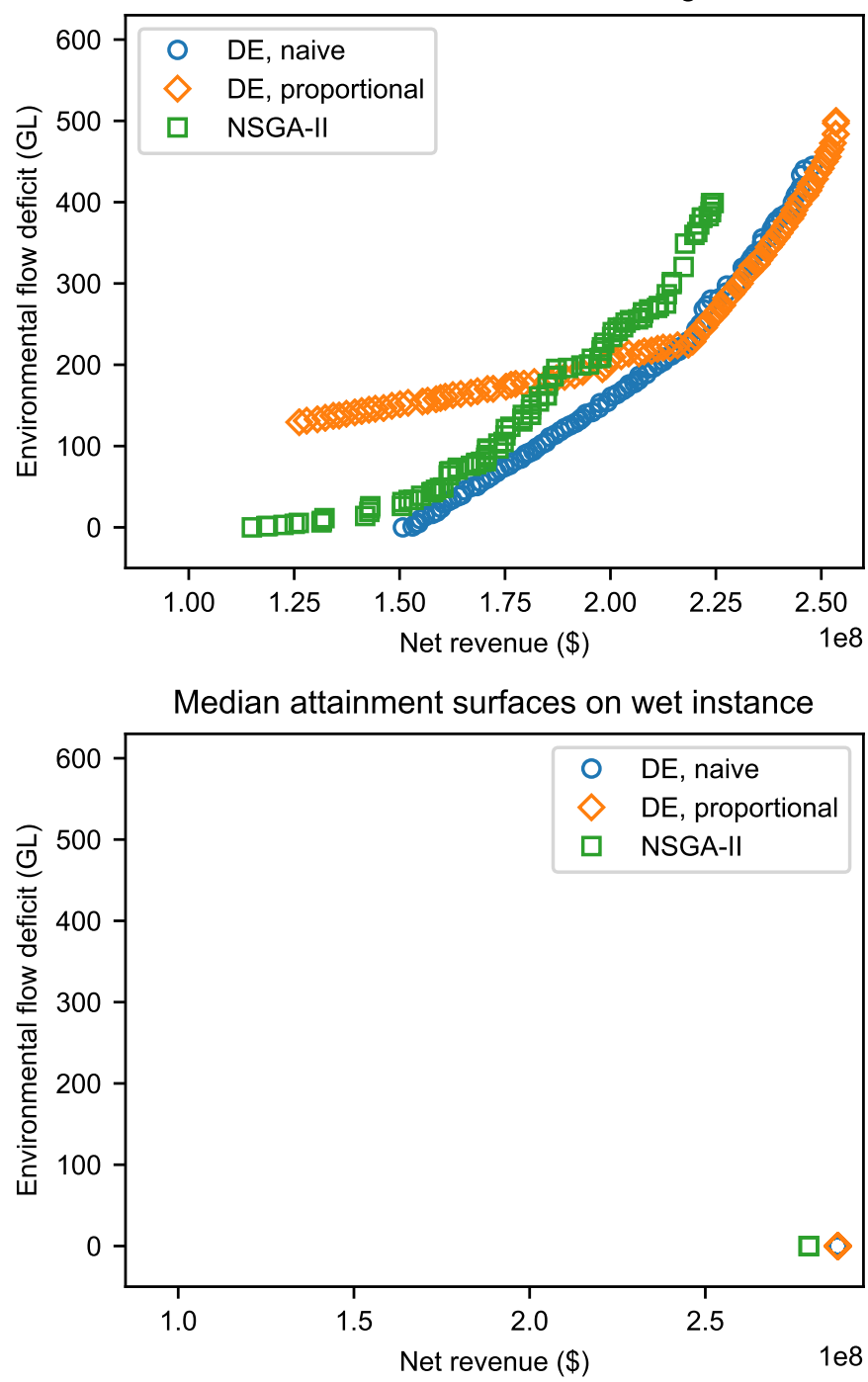



Best attainment surfaces on average instance
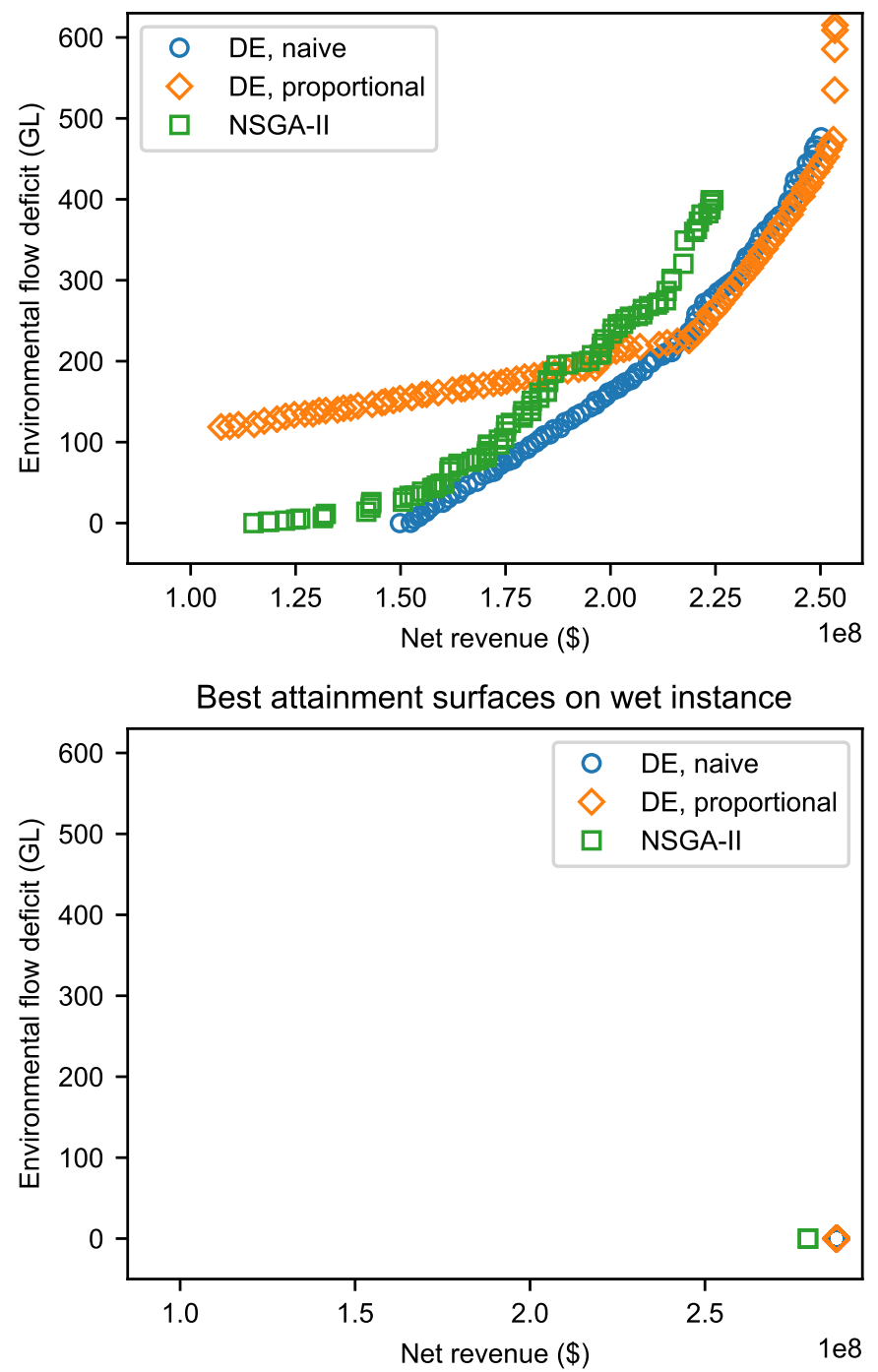

Fig. 4. Sample attainment surfaces for the dry, average and wet instances (top to bottom) produced by different approaches. The left column shows DE attainment surfaces with the median HV, while results for best HV are shown on the right. Only a single attainment surface is available for the NSGA-II, which is duplicated. The range and scale of the $\mathrm{x}$-axes vary by instance, given the highly different net revenue values possible under different rainfall conditions 
on the dry and average instances, it is clear that the two representations lead the algorithm to explore quite different regions of the search space, with the proportional representation able to produce solutions with higher net revenue at the expense of poorer environmental flows. Its attainment surfaces exhibit an obvious knee point at which its solutions (which must allocate the entire area) cannot compete with those of the naive representation in terms of environmental flow. This suggests that there may be some benefit in solving the problem using both representations and then selecting a single attainment surface from the two sets.

It is also evident that the DE results tend to be better than those produced by the NSGA-II approach. Given the relatively smooth surfaces produced by DE compared to the irregular surfaces produced by the NSGA-II, it is plausible that the NSGA-II would benefit from longer runs to continue to evolve solutions towards the Pareto front. This is also the case on the wet instance, where all three approaches eventually locate a single, globally dominant solution (within those result sets) with $E N=0$. With this instance the DE settled on a different mix of crops to the NSGA-II. NSGA-II retains some residual area given to rice, and a few other, vestigial allocations. This solution can be seen to be dominated by that found by DE, in which the total area has been devoted to cotton, resulting in a higher net revenue (although of very similar magnitude, $\$ 279.4 \mathrm{M}$ versus $\$ 287.6 \mathrm{M})$.

Figure 5 plots the attainment surfaces of the best trials for the dry and average instances and marks both extreme solutions and notable inflection points (in the case of DE proportional). The crop areas and proportion of fallow land for marked solutions are shown in Table I. Environmental flow details are not presented due to space limitations. However, water usage patterns by solutions with high net revenue are similar across algorithms, with lower environmental flow during the drier growing months of September through January, and the target flow generally met otherwise.

NSGA-II solutions appear to show greater diversity of crop mixes than those produced by $\mathrm{DE}$, which tends to specialise more. This may be one cause of the NSGA-II's poorer $N R$ performance, particularly evident on the selected wet instance solution and DE naive's solution A versus NSGA-II's solution $\mathrm{F}$ on the dry instance (in the second case, DE achieves a higher profit despite planting only $38 \%$ of the available space, compared to $58 \%$ by NSGA-II).

For DE proportional, the knee point appears to occur as it transitions from planting predominantly cotton to predominantly canola (consider solutions $\mathrm{D}$ and $\mathrm{E}$ on dry and $\mathrm{M}$ and $\mathrm{N}$ and average). Other key inflection points (solutions $\mathrm{K}$ and $\mathrm{L}$ on average) in dominated space where the algorithm is forced, due to the solution representation, to plant the entire area, are accompanied by greater crop diversity as it attempts to balance water requirements and constraints.

\section{CONCLUSION}

The use of limited water supplies in order to grow a variety crops while respecting environmental needs is a problem faced all around the world. High performance planning (i.e., optimisation) techniques are therefore necessary. In this paper we demonstrated that $\mathrm{DE}$ is able to find novel solutions to a multiobjective crop planning and water management problem, in a relatively modest number of solution evaluations. The two alternative solution representations allow the algorithm to produce differing solution sets, which demonstrate that:

- restricting the search space to those solutions that plant the entire area allows highly profitable (but less environmentally friendly) solutions to be discovered; but

- some land must be left fallow if environmental targets are to be approached while still maximising achievable profit.

As mentioned above, the model presented here only considers one year of planting (i.e., land is reserved for crops at the start of the year and not changed). However, farmers need to plan for extended timeframes: multiple years and sometimes decades. This necessitates changing crops and land allocations over time. Therefore, the next step of our research is to extend the present model with a temporal component. Finally, given changing climate conditions, this problem is a suitable candidate for robust optimisation techniques that solve for a variety of future rainfall scenarios, rather than treating these as independent problem instances.

\section{REFERENCES}

[1] M. Falkenmark, M. Finlayson, and L. Gordon, "Agriculture, water, and ecosystems: avoiding the costs of going too far," in Water for food, water for life: A comprehensive assessment of water management in agriculture. London, UK: Earthscan, 2007, pp. 234-277.

[2] E. Xevi and S. Khan, "A multi-objective optimisation approach to water management," Journal of Environmental Management, vol. 77, no. 4, pp. 269-277, 2005.

[3] A. Lewis and M. Randall, "Solving multi-objective water management problems using evolutionary computation," Journal of Environmental Management, vol. 23, pp. 179-188.

[4] K. Deb, Multi-Objective Optimization using Evolutionary Algorithms. Wiley, 2002.

[5] K. Price, "An introduction to differential evolution," in New Ideas in Optimization, D. Corne, M. Dorigo, and F. Glover, Eds. McGraw Hill, 1999, pp. 79-108.

[6] K. Price, R. Storn, and J. Lampinen, Differential Evolution: A Practical Approach to Global Optimization. Springer, 2005.

[7] A. Lewis and M. Randall, "Solving multi-objective water management problems using evolutionary computation," Journal of environmental management, vol. 204, pp. 179-188, 2017.

[8] P. B. Kumar, "Groundwater trading and management of local impacts Lower Murrumbidgee Deep Groundwater Source - 2013," NSW Office of Water, Sydney, Tech. Rep., 2013.

[9] K. Price, R. Storn, and J. Lampinen, Differential Evolution: A Practical Approach to Global Optimization. Berlin: Springer, 2005.

[10] J. Kennedy and R. C. Eberhart, "Particle swarm optimization," in IEEE International Conference on Neural Networks, Perth, Australia, 1995, pp. 1942-1948.

[11] J. Montgomery, M. Randall, and A. Lewis, "Differential evolution for RFID antenna design: A comparison with ant colony optimisation," in GECCO 2011, Dublin, Ireland, 2011, pp. 673-680.

[12] E. Zitzler and L. Thiele, "Multiobjective optimization using evolutionary algorithms - A comparative case study," in Fifth International Conference on Parallel Problem Solving from Nature (PPSN-V), A. E. Eiben, T. Bäck, M. Schoenauer, and H. P. Schwefel, Eds., Berlin, Germany, June 1998, pp. 292-301. 

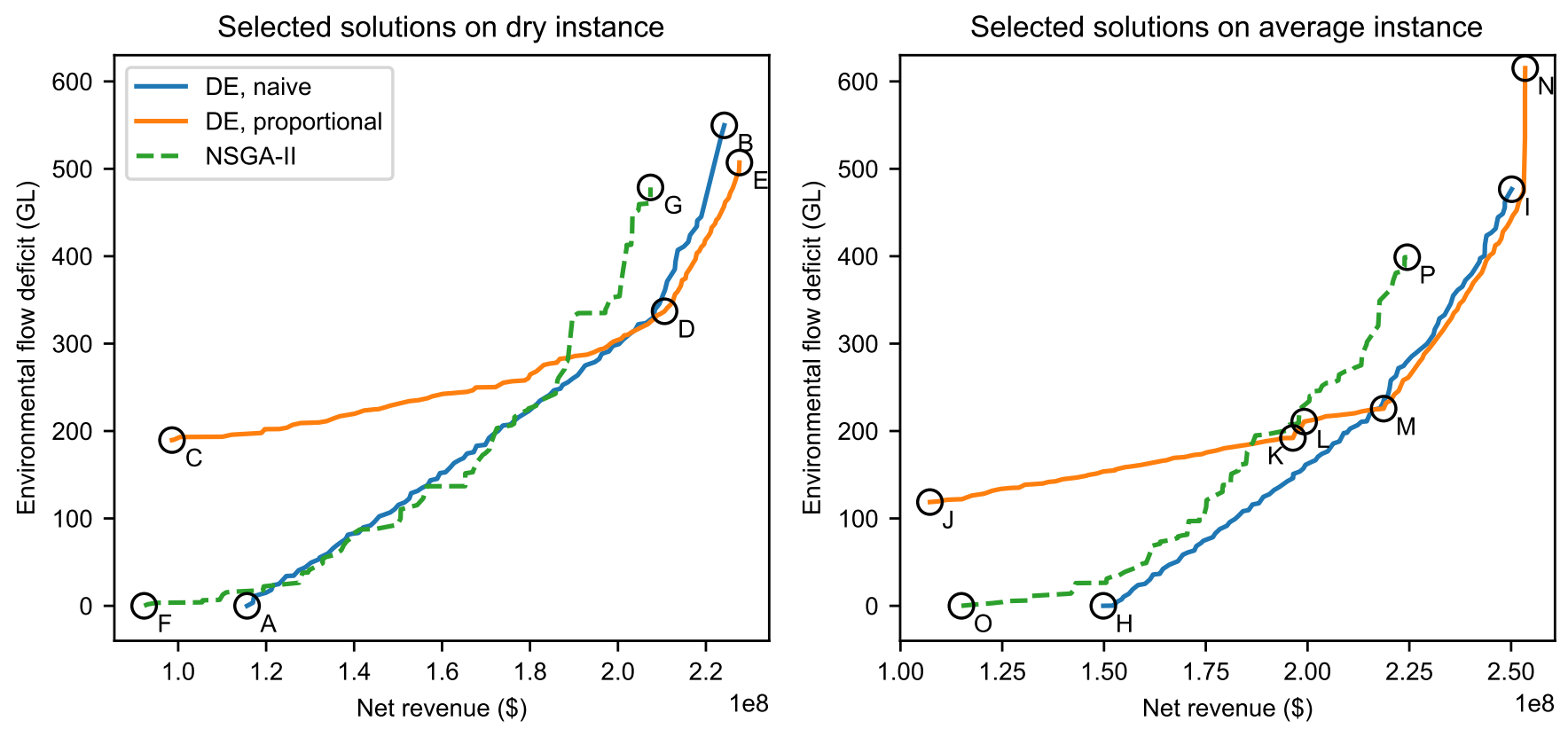

Fig. 5. Sample solutions whose crop areas are shown in Table I

TABLE I

SELECTED SOLUTIONS FROM ALGORITHMS' ATTAINMENT SURFACES

\begin{tabular}{|c|c|c|c|c|c|c|c|c|c|c|c|c|c|c|c|c|c|c|}
\hline ID & Alg. & $\mathrm{R}$ & W & B & M & $\mathrm{Ca}$ & $\mathrm{O}$ & S & WP & SP & $\mathrm{L}$ & Co & V & WV & SV & $\mathrm{Ci}$ & SF & fallow \\
\hline \multicolumn{19}{|c|}{ Dry instance } \\
\hline A & DE naive & - & 703 & 99 & - & $9.9 \mathrm{k}$ & 224 & 8 & 76 & - & 67 & $30.5 \mathrm{k}$ & - & $2.3 \mathrm{k}$ & $1.3 \mathrm{k}$ & $1.5 \mathrm{k}$ & 177 & $62 \%$ \\
\hline B & DE naive & - & 371 & 143 & - & $63.4 \mathrm{k}$ & 544 & - & 144 & - & 17 & $51.0 \mathrm{k}$ & 73 & $2.3 \mathrm{k}$ & $1.4 \mathrm{k}$ & $1.5 \mathrm{k}$ & 685 & $0 \%$ \\
\hline $\mathrm{D}$ & DE prop. & 452 & 17 & 1 & 7 & $83.6 \mathrm{k}$ & - & - & - & - & 27 & $32.5 \mathrm{k}$ & - & $2.3 \mathrm{k}$ & $1.4 \mathrm{k}$ & $1.5 \mathrm{k}$ & - & $0 \%$ \\
\hline E & DE prop. & - & - & - & - & $62.3 \mathrm{k}$ & - & 4 & - & - & - & $54.3 \mathrm{k}$ & - & $2.3 \mathrm{k}$ & $1.4 \mathrm{k}$ & $1.5 \mathrm{k}$ & - & $0 \%$ \\
\hline $\mathrm{F}$ & NSGA-II & $5.5 \mathrm{k}$ & $17.0 \mathrm{k}$ & 716 & $6.7 \mathrm{k}$ & $10.4 \mathrm{k}$ & $1.2 \mathrm{k}$ & $5.4 \mathrm{k}$ & 274 & 637 & - & $6.9 \mathrm{k}$ & $11.8 \mathrm{k}$ & $2.1 \mathrm{k}$ & $1.3 \mathrm{k}$ & $1.1 \mathrm{k}$ & 36 & $42 \%$ \\
\hline G & NSGA-II & $11.0 \mathrm{k}$ & $26.6 \mathrm{k}$ & 427 & 23 & $13.7 \mathrm{k}$ & $10.3 \mathrm{k}$ & - & 465 & $1.4 \mathrm{k}$ & - & $51.7 \mathrm{k}$ & - & $2.2 \mathrm{k}$ & $1.3 \mathrm{k}$ & $1.4 \mathrm{k}$ & 61 & $1 \%$ \\
\hline $\mathrm{H}$ & DE naive & - & $4.6 \mathrm{k}$ & - & 85 & $18.8 \mathrm{k}$ & - & 72 & - & - & - & $39.8 \mathrm{k}$ & - & $2.3 \mathrm{k}$ & $1.4 \mathrm{k}$ & $1.4 \mathrm{k}$ & - & $44 \%$ \\
\hline I & DE naive & - & - & 80 & 56 & $37.6 \mathrm{k}$ & 2 & - & - & - & - & $77.9 \mathrm{k}$ & - & $2.3 \mathrm{k}$ & $1.4 \mathrm{k}$ & $1.4 \mathrm{k}$ & 844 & $0 \%$ \\
\hline J & DE prop. & - & $15.5 \mathrm{k}$ & 316 & 590 & $7.5 \mathrm{k}$ & $44.5 \mathrm{k}$ & $33.0 \mathrm{k}$ & - & - & 93 & 202 & $15.0 \mathrm{k}$ & $2.3 \mathrm{k}$ & $1.4 \mathrm{k}$ & $1.5 \mathrm{k}$ & - & $0 \%$ \\
\hline $\mathrm{K}$ & DE prop. & $1.1 \mathrm{k}$ & 12 & 28 & 47 & $69.4 \mathrm{k}$ & 86 & 1 & 34 & 129 & - & $29.8 \mathrm{k}$ & $15.0 \mathrm{k}$ & $2.3 \mathrm{k}$ & $1.4 \mathrm{k}$ & $1.5 \mathrm{k}$ & 1000 & $0 \%$ \\
\hline $\mathrm{L}$ & DE prop. & 617 & $1.9 \mathrm{k}$ & 372 & $1.6 \mathrm{k}$ & $57.4 \mathrm{k}$ & $15.6 \mathrm{k}$ & $4.1 \mathrm{k}$ & 30 & 764 & 231 & $34.0 \mathrm{k}$ & - & $2.3 \mathrm{k}$ & $1.4 \mathrm{k}$ & $1.5 \mathrm{k}$ & - & $0 \%$ \\
\hline M & DE prop. & 1 & 16 & - & 24 & $76.3 \mathrm{k}$ & - & - & 1 & 17 & - & $40.3 \mathrm{k}$ & - & $2.3 \mathrm{k}$ & $1.4 \mathrm{k}$ & $1.5 \mathrm{k}$ & - & $0 \%$ \\
\hline $\mathrm{N}$ & DE prop. & - & 2 & 1 & 10 & $35.1 \mathrm{k}$ & - & - & - & 8 & - & $81.5 \mathrm{k}$ & - & $2.3 \mathrm{k}$ & $1.4 \mathrm{k}$ & $1.5 \mathrm{k}$ & - & $0 \%$ \\
\hline & DE prop. & - & - & - & - & - & - & - & - & - & - & $116.7 \mathrm{k}$ & - & $2.3 \mathrm{k}$ & $1.4 \mathrm{k}$ & $1.5 \mathrm{k}$ & - & $0 \%$ \\
\hline & NSGA-II & $13.2 \mathrm{k}$ & 169 & 4 & - & - & - & 1 & - & 15 & 6 & $103.4 \mathrm{k}$ & 6 & $2.2 \mathrm{k}$ & $1.4 \mathrm{k}$ & $1.4 \mathrm{k}$ & - & $0 \%$ \\
\hline
\end{tabular}

Crops are (R)ice, (W)heat, (B)arley, (M)aize, $(\mathrm{Ca})$ nola, $(\mathrm{O})$ ats, (S)oybean, winter pasture (WP), summer pasture (SP), (L)ucerne, (Co)tton, (V)ines, winter vegetables (WV), summer vegetables (SV), (Ci)trus and stone fruit (SF) 